\title{
RESEARCH
}

Open Access

\section{Histological correlates of postmortem ultra- high-resolution single-section MRI in cortical cerebral microinfarcts}

Deniz Yilmazer-Hanke ${ }^{1 *+} \mathbb{D}$, Theresa Mayer ${ }^{1}$, Hans-Peter Müller ${ }^{2}$, Hermann Neugebauer $^{2}$, Alireza Abaei ${ }^{3,4}$, Angelika Scheuerle ${ }^{5}$, Joachim Weis ${ }^{6}$, Karin M. E. Forsberg ${ }^{7}$, Katharina Althaus ${ }^{2}$, Julia Meier ${ }^{2}$, Albert C. Ludolph², Kelly Del Tredici', Heiko Braak', Jan Kassubek ${ }^{2 \dagger}$ and Volker Rasche ${ }^{3,4+}$

\begin{abstract}
The identification of cerebral microinfarctions with magnetic resonance imaging (MRI) and histological methods remains challenging in aging and dementia. Here, we matched pathological changes in the microvasculature of cortical cerebral microinfarcts to MRI signals using single $100 \mu$ m-thick histological sections scanned with ultra-highresolution 11.7T MRI. Histologically, microinfarcts were located in superficial or deep cortical layers or transcortically, compatible with the pattern of layer-specific arteriolar blood supply of the cerebral cortex. Contrary to acute microinfarcts, at chronic stages the core region of microinfarcts showed pallor with extracellular accumulation of lipofuscin and depletion of neurons, a dense meshwork of collagen 4-positive microvessels with numerous string vessels, CD68-positive macrophages and glial fibrillary acidic protein (GFAP)-positive astrocytes. In MRI scans, cortical microinfarcts at chronic stages, called chronic cortical microinfarcts here, gave hypointense signals in T1-weighted and hyperintense signals in T2-weighted images when thinning of the tissue and cavitation and/or prominent iron accumulation were present. Iron accumulation in chronic microinfarcts, histologically verified with Prussian blue staining, also produced strong hypointense T2*-weighted signals. In summary, the microinfarct core was occupied by a dense microvascular meshwork with string vessels, which was invaded by macrophages and astroglia and contained various degrees of iron accumulation. While postmortem ultra-high-resolution single-section imaging improved MRI-histological matching and the structural characterization of chronic cortical cerebral microinfarcts, miniscule microinfarcts without thinning or iron accumulation could not be detected with certainty in the MRI scans. Moreover, string vessels at the infarct margin indicate disturbances in the microcirculation in and around microinfarcts, which might be exploitable in the diagnostics of cortical cerebral microinfarcts with MRI in vivo.
\end{abstract}

Keywords: Post-mortem magnetic resonance imaging, Histological matching, Microbleeds, String vessels, Cerebral microangiopathy, Small vessel disease

\footnotetext{
* Correspondence: deniz.yilmazer-hanke@uni-ulm.de

†Deniz Yilmazer-Hanke, Jan Kassubek and Volker Rasche contributed equally to this work.

${ }^{1}$ Clinical Neuroanatomy, Department of Neurology, Institute for Biomedical Research, Ulm University, Helmholtzstr. 8/1, 89081 Ulm, Germany

Full list of author information is available at the end of the article
}

C C The Author(s). 2020 Open Access This article is licensed under a Creative Commons Attribution 4.0 International License, which permits use, sharing, adaptation, distribution and reproduction in any medium or format, as long as you give appropriate credit to the original author(s) and the source, provide a link to the Creative Commons licence, and indicate if changes were made. The images or other third party material in this article are included in the article's Creative Commons licence, unless indicated otherwise in a credit line to the material. If material is not included in the article's Creative Commons licence and your intended use is not permitted by statutory regulation or exceeds the permitted use, you will need to obtain permission directly from the copyright holder. To view a copy of this licence, visit http://creativecommons.org/licenses/by/4.0/ The Creative Commons Public Domain Dedication waiver (http://creativecommons.org/publicdomain/zero/1.0/) applies to the data made available in this article, unless otherwise stated in a credit line to the data. 


\section{Introduction}

Cerebral microinfarcts are found in the brains of elderly, in cognitive impairment and dementia of vascular origin, and in patients with various neurodegenerative diseases such as Alzheimer's or Parkinson's disease [2, 12, 52]. Despite growing evidence for the contribution of cortical cerebral microinfarcts to the development of cognitive deficits and dementia, their identification in conventional magnetic resonance imaging (MRI) remains challenging $[9,21,26,29,42]$. Owing to their small size, cortical microinfarcts often remain below the detection limit of the image resolution in conventional MRI at 1.5 $\mathrm{T}$ and $3 \mathrm{~T}$, although the detection rate of microinfarcts was improved in vivo with 7 T MRI $[28,45$, 48]. Furthermore, the large size of the human brain makes the histological sampling and detection of cerebral microinfarcts difficult in routine paraffin sections $[12,48]$.

Postmortem magnetic resonance imaging (MRI) can help to overcome the obstacles encountered in the detection of structural changes in the brain with in vivo MRI, because it allows the use of higher field strengths and longer acquisition times with a better image resolution and enhanced signal-to-noise ratio $[19,36,41]$. Although the detection of cortical cerebral microinfarcts could be improved using postmortem $7 \mathrm{~T}$ MRI, artefacts still caused false positive results and some histologically verified microinfarcts remained undetectable in MRI scans even upon re-evaluation of the images $[46,49]$. Currently it is unknown whether these cortical cerebral microinfarcts escaped detection and produced false negative results in MRI scans due to problems associated with technical limitations in image resolution at $7 \mathrm{~T}$ or MRI-histological matching [1,37], or whether there is a subset of microinfarcts that produces a different MRI signal pattern.

The aim of this study, therefore, was to correlate structural changes in cortical cerebral microinfarcts at both MRI and histological level. For this purpose, we first performed a thorough analysis of morphological features of cortical cerebral microinfarcts by focusing on their shape and location, their iron load, and patterns of microvascular anomalities in thick brain sections. To overcome challenges that result from histological matching of microinfarcts to MRI scans [1], in a next step we developed a new ultra-highresolution postmortem MRI technique by imaging single thick histological sections with proven microinfarcts with MRI at 11.7 T. Using this approach, we then compared MR signals produced by microinfarcts directly to morphological correlates of microinfarcts by staining the MR-imaged sections with various histological techniques. For a general histopathological characterization of microinfarcts, sections were stained with methods recently developed in our laboratory that visualize extracellular lipofuscin granules indicative of neuronal cell death, aldehyde fuchsine-positive macrophages, and alterations in the capillary network of microinfarcts [8]. MRI signals obtained in microinfarcts were further correlated with iron histochemistry and other histological alterations in MRI-scanned and adjacent sections. Microvascular changes and glial pathology were visualized using double-label immunohistochemistry for collagen together with markers of the vascular endothelium, astroglia or macrophages [22].

\section{Material and methods}

Subjects and neuropathological evaluation of the brain

Tissue from autopsy cases preserved at the Ulm University Tissue Bank underwent routine neuropathological examination. Thick brain sections used for diagnostics were screened for microinfarcts. Ten cases with cortical cerebral microinfarctions ( 5 females and 5 males, age range 69-89 years) were randomly selected for the study (for details: Table 1). Cases in the study cohort studied had Alzheimer's disease (AD)-related argyrophilic neurofibrillary changes (NFT) at limbic stage 3 or less except case 8 (89 year old female with mixed dementia at NFT stage 4, MMSE 15/30, CDR 1.0) and varying degrees of extracellular $\mathrm{A} \beta$ deposition at cortical amyloid stages 0 (three cases) to A-C (seven cases) [6]. Staging of neuronal alphasynuclein pathology [7] indicated Parkinson's disease (PD) stage 0 in all cases except in case 10 (74 year old female at Parkinson's stage 4). None of the cases were diagnosed with other tauopathies or alpha-synucleinopathies. This retrospective study was conducted in compliance with the university ethics committee guidelines as well as German federal and state law governing human tissue usage and in accordance with the Declaration of Helsinki. Informed written permission was obtained from all patients and/or their next of kin for autopsy.

\section{Histological processing and diagnostics}

Brains were fixed in a $4 \%$ solution of formaldehyde and cut in approximately $1 \mathrm{~cm}$ thick coronal slices. Tissue slices containing frontal, mid-hemispheric, occipital and cerebellar blocks as well as blocks of the brainstem (rostral medulla, pontine-mesencephalic junction and midbrain) were embedded in polyethylene glycol (PEG 1000, Merck, Carl Roth Ltd., Karlsruhe, Germany). In addition, multiple coronal hemispheric blocks were available in 2 cases (Table 1). Several $100 \mu \mathrm{m}$ thick consecutive sections were cut from each block with the aid of a sliding microtome (Jung, Heidelberg, Germany). Alzheimer-related neurofibrillary changes and extracellular deposits of $A \beta$ peptide were visualized with advanced silver staining methods $[6,23]$ and immunohistochemistry with the mouse anti-phospho-tau (Ser202, Thr205) antibody (1.2000, clone AT8, Fisher Scientific $\mathrm{GmbH}$, Schwerte, Germany) and mouse anti- $\beta$-amyloid (A $\beta$ ) 17-24 antibody (1:5000, clone 4G8, BioLegend, Koblenz, Germany). Alpha-synuclein pathology was detected 


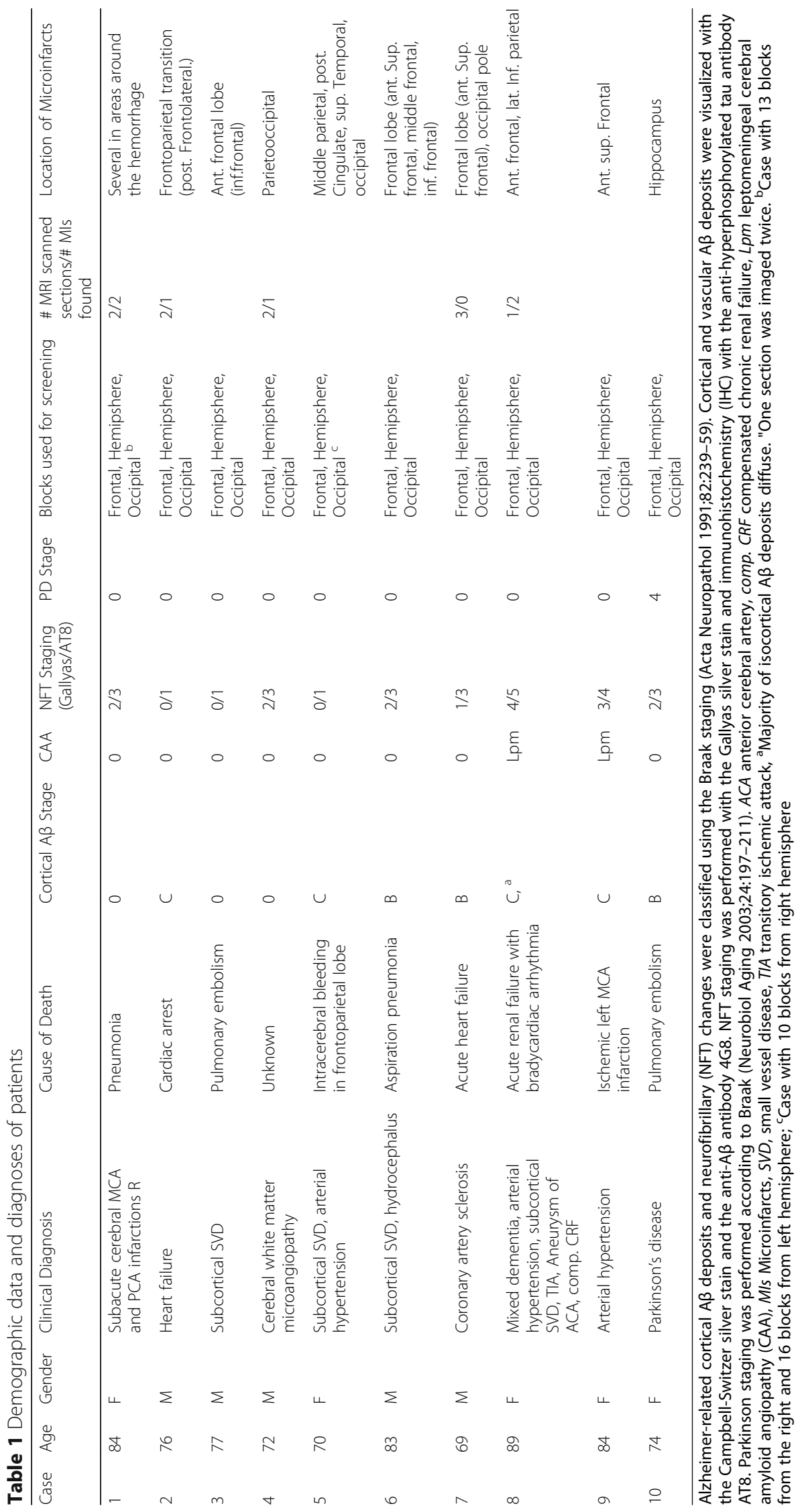


with the anti-syn-1 antibody (1:2000, clone number 42; BD Biosciences, CA, USA).

For screening of microinfarcts, sections obtained from each coronal block were stained with the pigment Nissl stain (PN) using aldehyde fuchsine and Darrow red [4] in all cases. Additional sections stained with a modified hematoxylin eosin (H\&E) procedure [22] were available in 8 cases (sections from all blocks in cases $1 \& 5 ; 12$ sections in the other 6 cases), and iron accumulation was visualized with Prussian blue staining [35] in 5 cases (16 sections). Free-floating sections adjacent to sections with proven microinfarcts were selected for MRI scanning and further histological analysis.

\section{Single section ultra-high resolution MR imaging}

Magnetic resonance (MR) images were taken at room temperature on an $11.7 \mathrm{~T}$, horizontal bore $(160 \mathrm{~mm}$ diameter) dedicated small animal system (BioSpec 117/16, Bruker Biospin, Ettlingen, Germany) equipped with BGA9 shielded gradients. The signal was recorded with a $72 \mathrm{~mm}$ quadrature volume transmit/receive resonator. Before imaging, the $100 \mu \mathrm{m}$-thick brain sections were stretched on glass object slides and coverslipped in a $0.9 \% \mathrm{NaCl}$ saline solution by paying attention to minimize trapping of air bubbles (Fig. 1). Next, object slides with the sections were sealed tightly to prevent penetration of air or external solutions to the zone around the sections or to cavitated areas within the sections. The sealed object slides were placed in a custom-built Plexiglas chamber. The chamber was degased and filled with Fomblin (Sigma, St Louis, MO, USA). The samples were placed in the iso-center of the magnet. Multi-contrast three- dimensional (3D) data was obtained, including: T1-weighted $(\mathrm{TR}=500, \mathrm{TE}=11.5), \mathrm{T} 2$-weighted $(\mathrm{TR}=3500, \mathrm{TE}=45)$ and proton density $(\mathrm{PD}, \mathrm{TR}=2000, \mathrm{TE}=8.5)$ single spin echo images with one signal average $(\mathrm{NSA}=1)$ as well as gradient echo $\mathrm{T} 2 *$-weighted images $(\mathrm{TR}=24, \mathrm{TE}=12$, flip angle $=30^{\circ}$ ) with NSA $=16$. Spatial resolution was as $100 \times 110 \times 200 \mu \mathrm{m}^{3}$. The single section ultra-high-resolution MRI technique was first established by scanning 3 sections without microinfarcts. In a next step, 10 sections with microinfarcts from 5 cases were imaged with the MRI scanner. In one case (case 4), two neighboring sections from the same microinfarct were imaged. In another case (case 1), one of the sections was imaged twice, confirming the reproducibility of MRI signals produced by the same microinfarct (for details: Table 1).

\section{Immunohistochemistry (ICH)}

Sections imaged with MRI and adjacent sections (free-floating, $100 \mu \mathrm{m}$ thick) were first treated with $10 \%$ methanol and $3 \%$ concentrated $\mathrm{H}_{2} \mathrm{O}_{2}$ in Tris-buffered saline (TBS) and then with bovine serum albumin (BSA). Antigen retrieval was performed using Tris-EDTA buffer at $\mathrm{pH} 9.0$ or citrate buffer at $\mathrm{pH} 6.0$ for $1 / 2 \mathrm{~h}$ at $100^{\circ} \mathrm{C}$ or pretreatment with $1.3 \mu \mathrm{g} / \mathrm{ml}$ proteinase $\mathrm{K}$ for $10-15 \mathrm{~min}$ at $37^{\circ} \mathrm{C}$ (Invitrogen, Darmstadt, Germany). The sections were incubated with primary antibodies $\left(12-48 \mathrm{~h}, 4^{\circ} \mathrm{C}\right)$ against collagen 4 (COLL4; 1:5000, rabbit, Abcam, Cambridge, UK), CD68 (1:2000, mouse, DAKO, Glostrup, Denmark) or glial fibrillary acidic protein (GFAP; 1:1000, rabbit, Abcam, Cambridge, UK) and a secondary biotinylated antibody $(1: 200 ; 2 \mathrm{~h}$, room temperature, Vector

\section{Dissection of Area with Microinfarct}

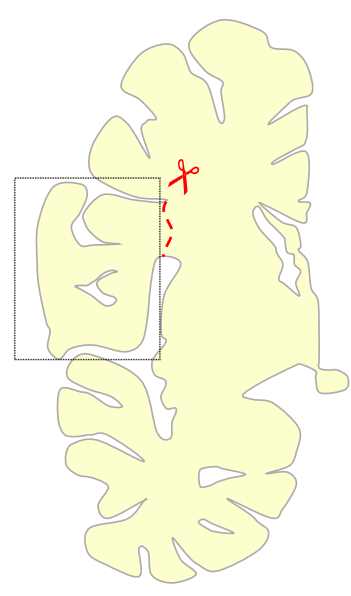

hemisphere section with microinfarct

\section{High-resolution MRI Scanning at $11.7 \mathrm{~T}$}

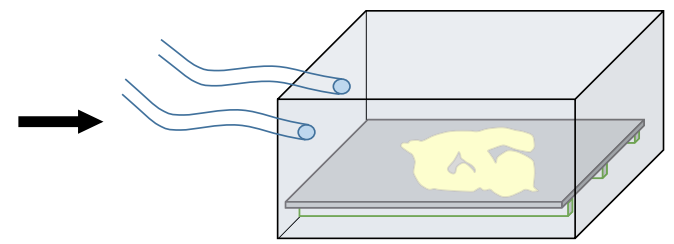

Degased Plexiglas chamber filled with Fomblin

$+$

dissected section with microinfarct, coverslipped with saline

\section{Histological Staining}

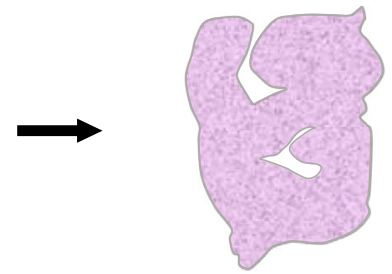

dissected section with microinfarct, stained

Fig. 1 Procedure used to scan individual free-floating $100 \mu \mathrm{m}$ thick sections with ultra-high-resolution MRI. The part of the section containing the microinfarct was dissected from the hemisphere section, coverslipped with saline, and imaged in a custom-made Plexiglas chamber in the MRI scanner. After completion of the scans, the section was processed for histological staining 
Laboratories, Burlingame, CA, USA). Alternatively, sections were incubated for $48 \mathrm{~h}$ with Ulex europaeus lectin (UEA-1; 1: 800, biotin-coupled, GeneTex, Irvine, CA, USA). Immunohistochemical reactions or lectin binding were visualized with an avidin-biotin-peroxidase complex (ABC Vectastain, Vector Laboratories, Burlingame, CA, USA) and 3,3'-diaminobenzidine tetrahydrochloride (DAB; Sigma Taufkirchen, Germany). For double-label immunohistochemistry, sections were washed with TBS at $95^{\circ} \mathrm{C}$ for $5 \mathrm{~min}$, and the procedure was repeated using the next primary and secondary antibody. Subsequently, a blue chromogen (Vector SK-4700 peroxidase substrate kit, Linaris, Doffenheim; Germany) was used to visualize the reaction product. Omission of the lectin or primary antibody resulted in lack of staining.

The Coll4-positive microvasculature was evaluated in all microinfarcts, which had been identified in neighboring $\mathrm{PN}$ and $\mathrm{H} \& \mathrm{E}$ stained sections (see Table 1). In addition, histological characteristics of microinfarcts were studied using double-label-IHC for Coll4 and GFAP ( 9 sections, in 6 cases), for Coll4 and CD68 (14 sections, in 6 cases), for Coll 4 and $A \beta$ (8 sections, in 7 cases) and for Coll4 and UEA-l (8 sections, 4 cases). MRI-scanned sections were stained using Coll4-/UEAdouble-labeling $(n=5)$, H\&E $(n=2)$, Prussian blue $(n=1)$, and Coll4-/CD68-double-label-IHC $(n=2)$.

\section{Image acquisition and processing}

Dicom images were viewed with the software RadiAnt DICOM Viewer (version 4.6.5) and Image J (version v1.52j) and exported in tiff-format. Brightness and contrast of images were optimized with the GNU Image Manipulation Program (GIMP - version 2.8.16) and Adobe Photoshop (version 10.0). The tissue object was segmented and presented on a black background for an optimal comparability of the different scan sequence contrasts. To visualize the microinfarct zone in selected areas and insets, images were re-scaled by cubic interpolation. Enlarged images were smoothened using Gaussian blurring and/or despeckling. Microphotographs of histological sections were taken with a digital camera (Jenoptik Progres Gryphax Prokyon, Jena, Thüringen, Germany) using an AX10 microscope (Zeiss, Jena, Germany). For the detection of lipofuscin autofluorescence, sections were imaged with the aid of a LED fluorescence lamp and narrow selective green $\mathrm{H}$ bandpass filter set (F46-801, AHF Analysetechnik, Tübingen, Germany). In IHC-stained sections, either single images were taken or z-stacks were obtained with bright field microscopy. Images were processed with the software Adobe Photoshop (version 10.0) to optimize light and contrast conditions as well as color temperature, hue and saturation. Multiple single images and $z$-stacks were stitched to visualize larger areas or merged in the z-dimension as needed.

\section{Results \\ Chronic cortical cerebral microinfarcts contained extracellular pigment granules}

Cortical cerebral microinfarcts were found in cases with and without cortical $A \beta$ deposits, hence, also in the absence of cerebral amyloid angiopathy (CAA) (Table 1). Microinfarcts could be easily detected by screening the cerebral cortex in PN-stained sections [8]. The PN stain also helped to distinguish (sub)acute microinfarcts from microinfarcts at chronic stages. The latter microinfarcts, which were now called chronic cortical cerebral microinfarcts here, stood out in the PN stain through tissue pallor and lipofuscin pigment accumulation (Fig. 2a, c and e; Fig. 3a-b). In all chronic microinfarcts studied, lipofuscin granules formed disorganized aggregates outside the cytoplasm of Darrow red-stained neurons or other cells (Fig. 2c1 and e1). The disorganized lipofuscin granules were located in areas with neuronal cell loss showing pallor. Hence, they resembled extraneuronal lipofuscin remnants typically found in areas with extended neuronal cell loss in other neurological diseases (e.g., [5, 8, $16,54])$. The extracellular lipofuscin aggregates in microinfarcts could be clearly distinguished from intraneuronal lipofuscin granules (Fig. 2e2, see also [44]) or the purplish, cytoplasmic aldehyde fuchsine staining seen in macrophages that clustered in the microinfarct core (Fig. 2a1, see also [8]). In the H\&E stain adapted to thick sections, chronic microinfarcts also displayed pallor and tissue thinning (Fig. 4 and Supplementary file S1). However, pallor was mild compared to the PN stain in small cortical infarcts with minor tissue thinning, making it difficult to detect the microinfarction zone (Supplementary file S1). (Sub)acute ischemic cortical cerebral microinfarcts, by contrast, only exhibited pallor of the Nissl substance in the absence of extracellular lipofuscin accumulation (Fig. 5a, case 5, died shortly after admission to hospital).

\section{Chronic microinfarcts contained a dense microvascular meshwork with string vessels}

A dense meshwork of Coll4-positive microvessels was found in all microinfarcts that fulfilled the criteria of chronic microinfarcts in the PN stain as described above. The dense microvascular meshwork covered the heart of the microinfarction zone with tissue pallor, which contained extracellular lipofuscin aggregates (Fig. $2 \mathrm{a}_{2}, \mathrm{c}_{1}$ and $\mathrm{e}_{1}$ ) and/or macrophages (Fig. $2 \mathrm{a}_{1}$ and $\mathrm{e}_{1}$; see also [8]). The dense microvascular meshwork also occupied the "cavitated" zone of chronic microinfarcts with puckering and cavitation (Figs. 4c and 10e). Chronic microinfarcts further exhibited many Coll4-positive string vessels, which correspond to thin connective tissue strands that are formed by collapsed basement scaffolds connected to the capillary network [10, 22]. Especially, the microinfarction zone covered with the dense microvascular meshwork contained numerous Coll4-positive string vessels. Moreover, string vessels 


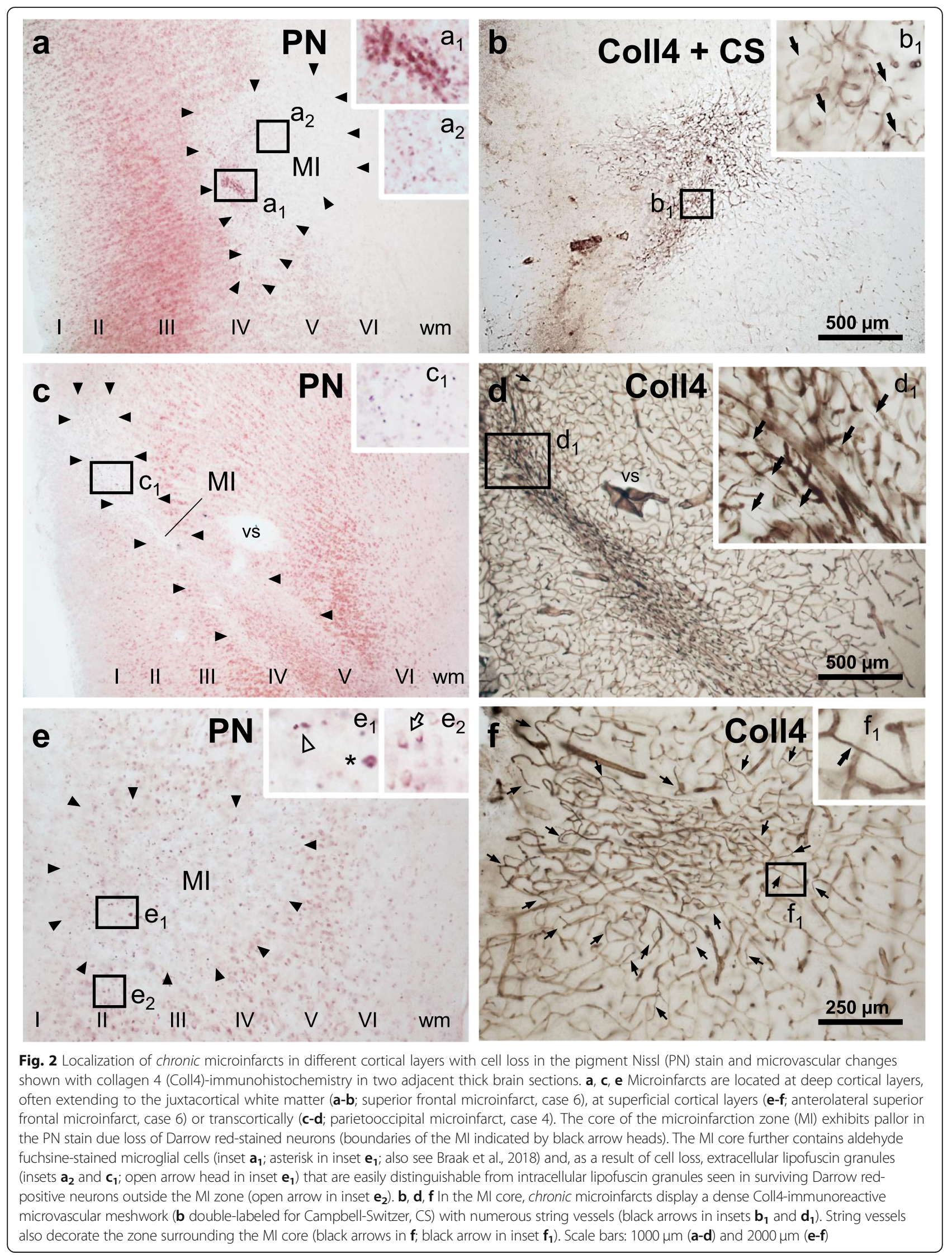




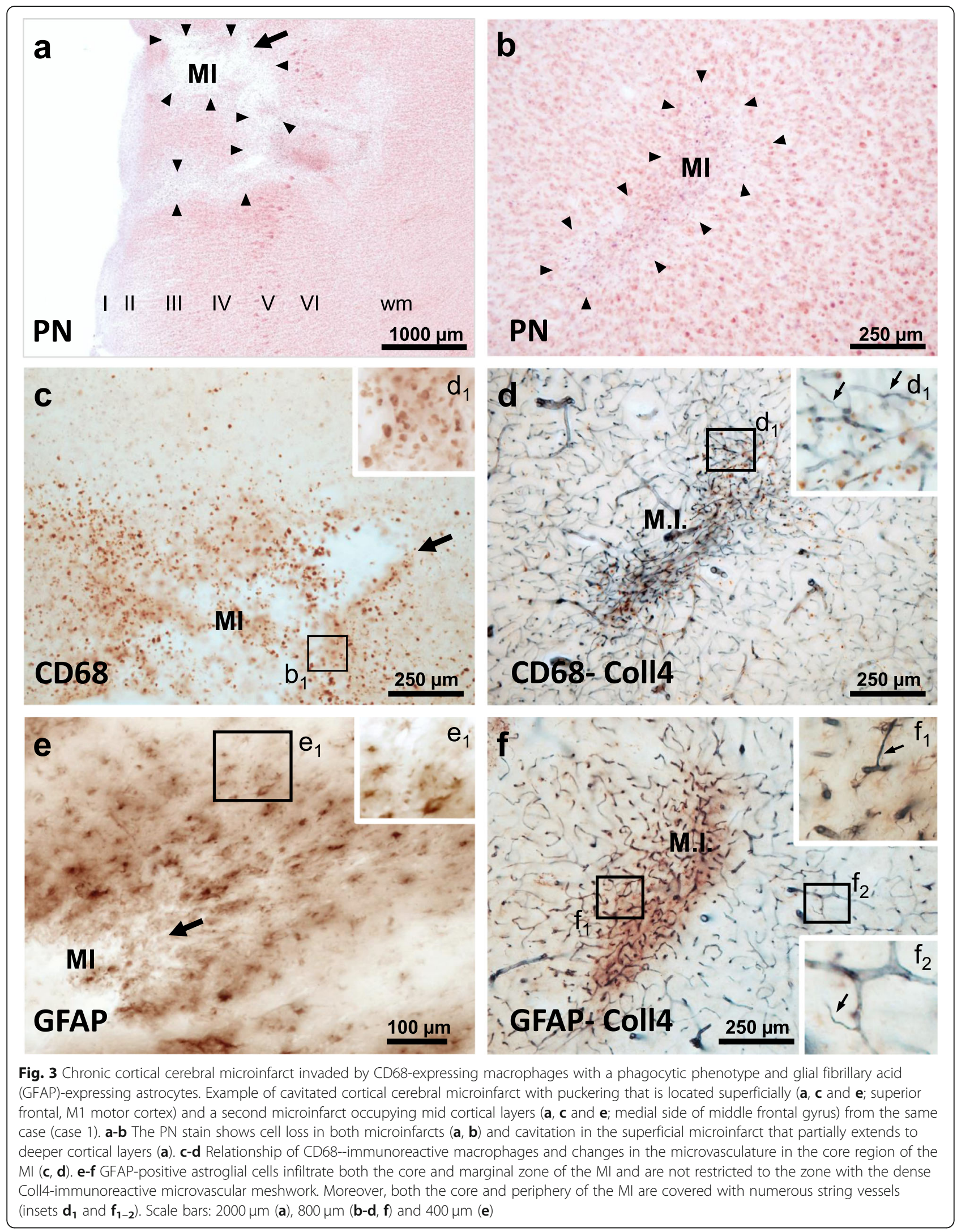



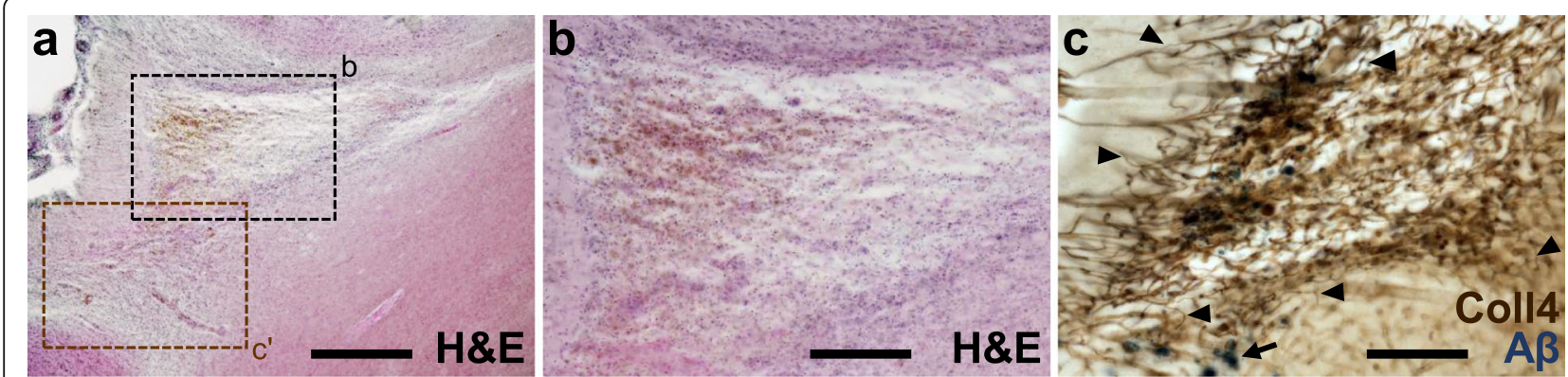

Fig. 4 Modified H\&E stain (case 5) showing a chronic cortical cerebral microinfarct with comparatively large tissue thinning and cavitation in the occipital lobe, which extends from mid-cortical layers to the juxtacortical zone (a-b). In a neighboring section double-labeled for Coll 4 and beta-amyloid (A $)$, the same microinfarct displayed a dense microvascular meshwork with numerous string vessels (arrow heads) and parenchymal A $\beta$ deposits (arrow) (c). The inset (c') in the H\&E-stained section (h) corresponds approximately to the same area as in (c). Scale bars: $500 \mu \mathrm{m}(\mathbf{a})$ and $200 \mu \mathrm{m}(\mathbf{b}-\mathbf{c})$

were observed in the marginal zone of microinfarcts, which surrounded the area with the dense microvascular meshwork (Figs. 2 and 3). In double-labeled sections, Coll4positive string vessels often lacked staining with the lectin UEA-l (see Fig. 10), which indicated endothelial cell damage, since UEA-1 binds to the glycocalyx on the luminal surface of vascular endothelial cells [22]. The dense microvascular meshwork with string vessels was also found in a cortical microinfarct with heavy iron accumulation, although this case exhibited diffuse cortical $A \beta$ deposits and no CAA (Fig. 6c-f). Such microvascular changes were absent in (sub)acute ischemic cortical cerebral microinfarcts (Fig. 5).

\section{Chronic microinfarcts are infiltrated by CD68- and GFAP- positive cells}

In all chronic cortical cerebral microinfarcts studied, the core of the infarction zone was invaded by numerous CD68-positive macrophages, which coincided with the area covered by the dense microvascular meshwork (Fig. 3c-d). The infarction zone of all chronic microinfarcts also contained a prominent astrocytic scar (Fig. 3e-f). Both cell types were not limited to the area with the highest microvascular density. They also covered the margin of microinfarcts and some cells extended to the neuropil surrounding the microinfarct. Moreover, astrocytes surrounding microinfarcts with cavitation appeared larger than astrocytes in microinfarcts with minor thinning of the tissue (Fig. 3e).

\section{Cortical cerebral microinfarctions occur in different cortical layers}

The size and location of the microinfarcts was determined using corresponding PN-stained and Coll4-labeled sections. The area that showed overlap of tissue pallor in the PN stain (Fig. 2a, c and e) and a dense Coll4-positive microvascular meshwork was defined as the microinfarction zone (Fig. 2b, $d$ and $\mathrm{f}$ ). Microinfarcts ranged from

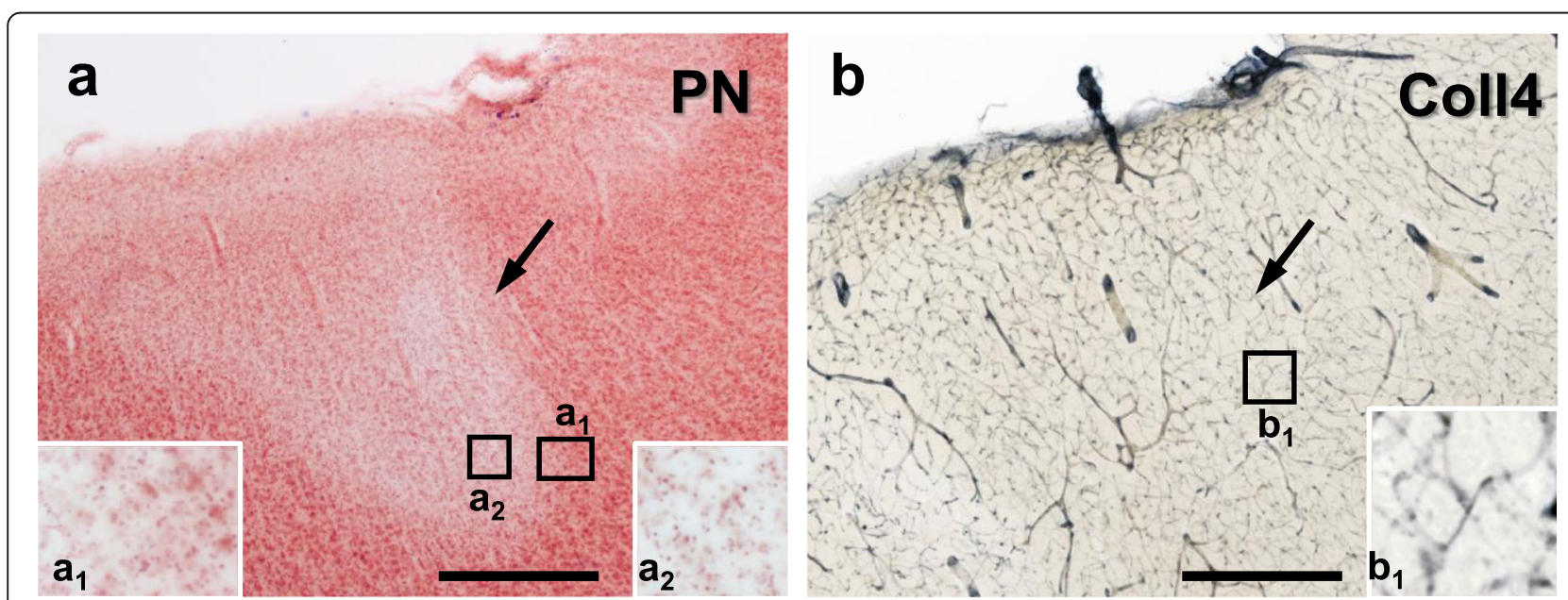

Fig. 5 Subacute superficial cortical cerebral microinfarct (case 5) with pallor in the PN stain (a). At higher magnification, neuronal somata show reduced Darrow red (Nissl) staining, but no extracellular lipofuscin granules are visible $\left(\mathbf{a}_{\mathbf{1}}\right.$ and $\left.\mathbf{a}_{\mathbf{2}}\right)$. Also, no string vessels or other obvious changes are evident in the microvascular network visualized with IHC using antibodies against Coll4 (b and inset $\mathbf{b}_{\mathbf{1}}$ ). Scale bars: $500 \mu \mathrm{m}$ (a-b) 

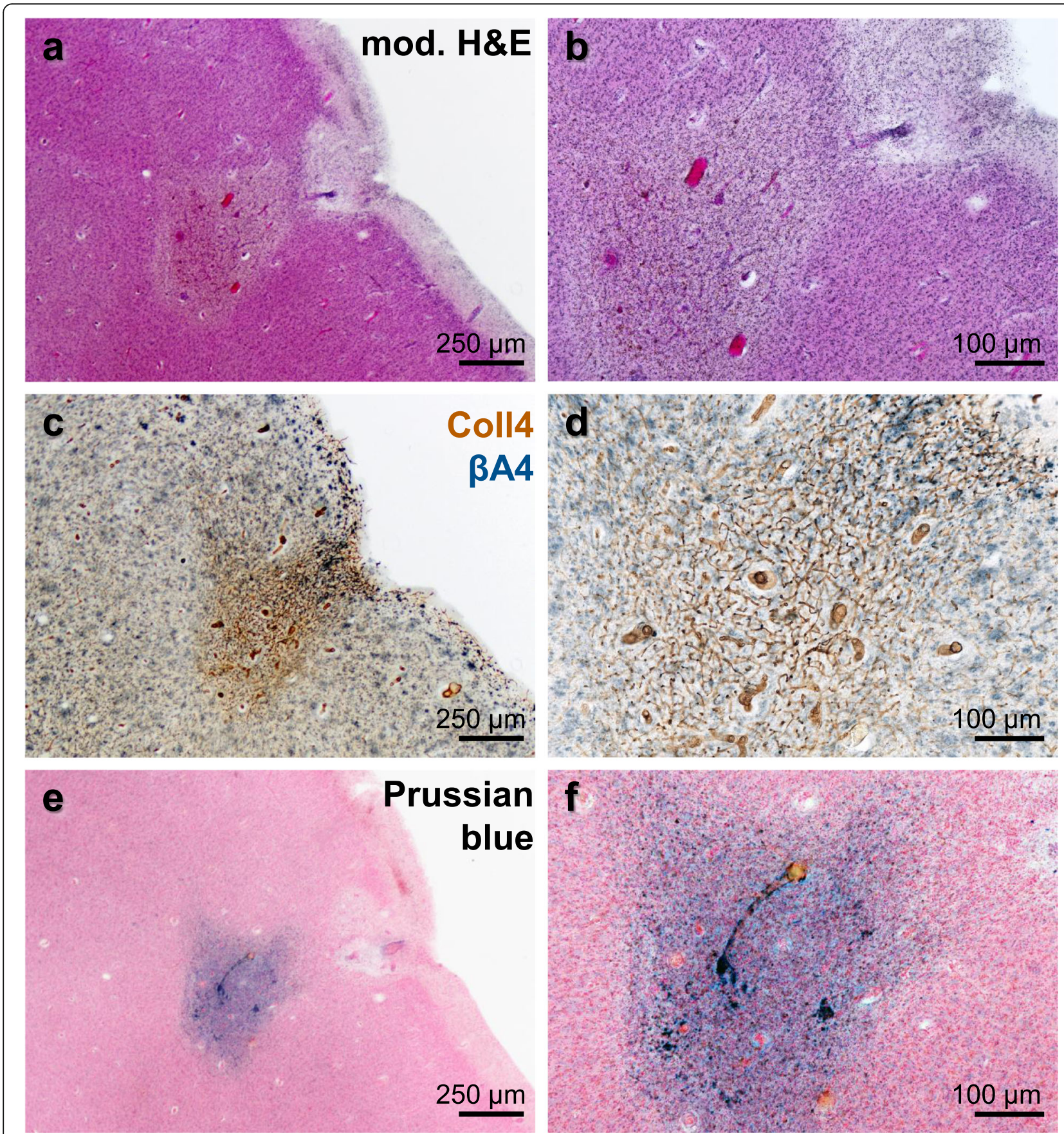

Fig. 6 Morphological characteristics of a superficial cortical microinfarct shown in three adjacent thick sections from a case with diffuse parenchymal $A \beta$ deposits (case 8). $\mathbf{a}-\mathbf{b}$ The microinfarction zone shows pallor and altered microvessels in the modified H\&E stain. $\mathbf{c}$-d Microvascular changes in this case consist of a dense microvascular meshwork with a high density of string vessel as seen in cases without $A \beta$ deposits. e-f Prussian blue staining shows iron accumulation in the core of the microinfarction zone. Scale bars: $250 \mu \mathrm{m}(\mathbf{a}, \mathbf{c}$ and $\mathbf{e})$ and $100 \mu \mathrm{m}$ $(\mathbf{b}, \mathbf{d}$ and $\mathbf{f}$ )

$200 \mu \mathrm{m}$ to $2000 \mu \mathrm{m}$ in width and/or length and were identified in all layers of the cerebral cortex (Supplementary file S2). Superficial and deep cortical microinfarcts were often broader, whereas transcortical microinfarcts were usually narrow. Superficial cortical microinfarcts frequently led to an indentation of the cortical surface. Deep infarctions with a juxtacortical position extended to the adjacent white matter (Fig. 2). Microinfarct locations were compatible with the layer-specific blood supply of the cerebral cortex by arterioles classified as A1 to A6 type 


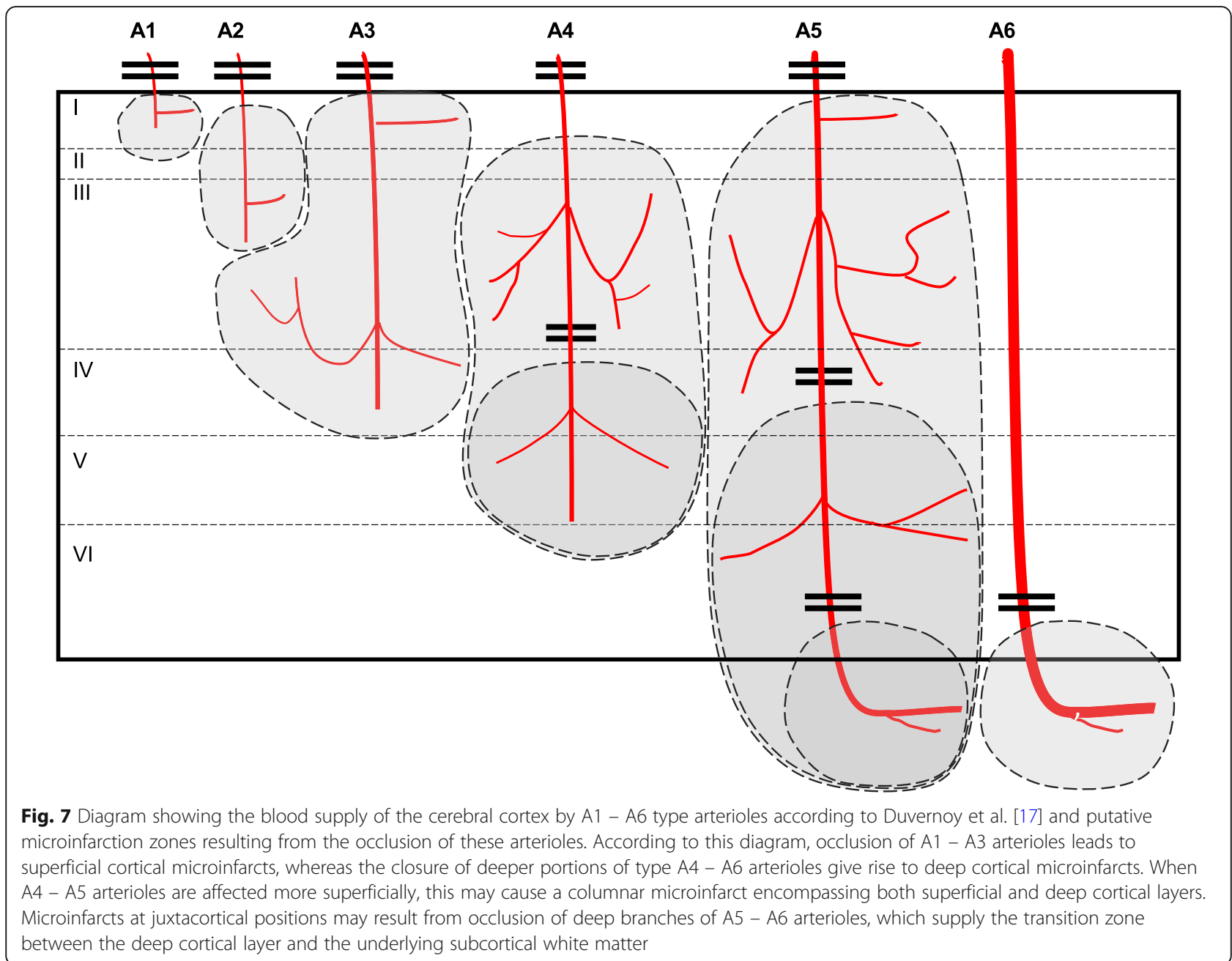

arterioles by Duvernoy and colleagues [17]. Moreover, some transcortical microinfarctions bifurcated in deeper cortical layers indicating that both the main arteriolar branch and its side branches were affected (Fig. 2c). Thus, a disturbance in the circulation in A1-4 arterioles (e.g., through hypoperfusion, occlusion or thromboembolic closure) might lead to microinfarcts in superficial and middle cortical layers, whereas a cessation of the circulation in A4-6 arterioles could lead to microinfarcts in deep cortical and juxtacortical layers, and a full disruption of A5 arterioles to transcortical microinfarcts (Fig. 7).

\section{Variability of single section ultra-high resolution MRI signal intensities among microinfarcts}

Altogether 6 histologically verified microinfarcts were identified in 5 MRI-scanned sections (Table 1). The microinfarct area, which showed pallor in the PN stain and was covered by a dense microvascular meshwork in the histological sections, produced hypointense T1- and PD-weighted MRI signals and hyperintense T2-weighted MRI signals. Moreover, in T2*-weighted gradient echo images, a hypointense signal with a blooming effect was seen in microinfarct zone, which probably originated from paramagnetic signals produced by iron aggregates. However, the intensity of MRI signals produced by the chronic cortical cerebral microinfarcts varied considerably, although in histological sections all microinfarcts exhibited similar pathological features, such as a dense Coll4positive microvascular meshwork with string vessels, loss of Nissl-stained neurons, and extracellular lipofuscin granules. The intensity of T1-weighted and PD-weighted MRI signals of microinfarcts ranged from low (Fig. 8; Supplementary files S3 and S4) to strong (Figs. 9 and 10). A stronger T1- or T2-weighted hypo-/hyperintense signal correlated with tissue thinning and cavitation (Fig. 10). In some microinfarcts, the rim of the microinfarction zone gave a slightly hyperintense MRI signal in PD-weighted images (Fig. 10c, Supplementary file S3). Furthermore, the intensity of T2\%-weighted MRI signals correlated more with the level of iron accumulation in the microinfarct core rather than the density of parenchymal diffuse or cored $\mathrm{A} \beta$ plaques. In $\mathrm{T} 2 \%$-weighted images, microinfarcts 

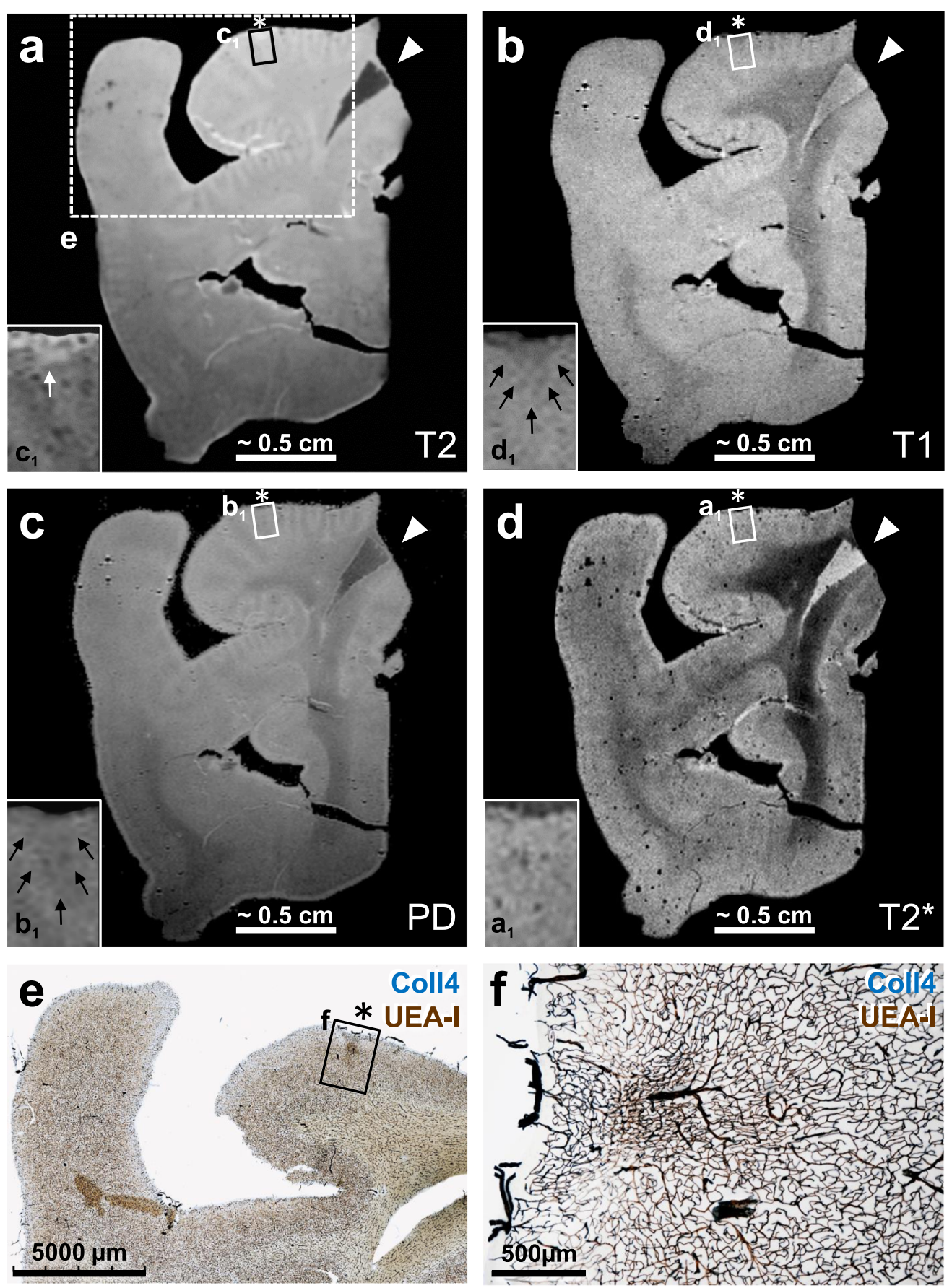

Fig. 8 Postmortem ultra-high-resolution MRI of a $100 \mu \mathrm{m}$ thick section scanned at $11.7 \mathrm{~T}$ followed by post-imaging histological staining (case 2). a The microinfarct (asterisk) could not be identified in the $T 2^{*}$-weighted image, and the microinfarction zone showed very little iron deposition as indicated by Prussian blue staining in an adjacent section (not shown). However, microvessels appeared enlarged due to the blooming effect, probably induced by susceptibility effects of iron magnetism in erythrocytes (see inset $\mathbf{a}_{\mathbf{1}}$ ). $\mathbf{b}$ Proton density (PD) image sequence reflecting the proton (water) content in the tissue also failed to visualize this microinfarct (see asterisk and inset $\mathbf{b}_{1}$ ). $\mathbf{c}$ In the T2-weighted image, a slightly enhanced hyperintense signal was observed at the superficial part of the infarction zone (white arrow in inset $\mathbf{c}_{\mathbf{1}}$ ). $\mathbf{d}$ The superficial zone of the microinfarct (see asterisk) also gave a slightly hypointense signal in the T1-weighted image (area indicated by black arrows in inset $\mathbf{d}_{\mathbf{1}}$ ). e-f The MRI-scanned section showed a microinfarct with characteristic changes in the microvasculature as seen in double-labeling for Coll4 and UEA-I lectin, which visualizes the endothelial glycocalyx, but the microinfarct area covered by the dense microvascular meshwork could not be distinguished well from the surrounding tissue based on MRI signals [see in frame $\mathbf{f}$ in (e) and the enlarged inset in (f)]. Arrow heads (a-d) points to an artefact resulting from an overlay of two flee-floating ends of the section. Scale bars: $3 \mathrm{~mm}(\mathbf{e})$ and $500 \mu \mathrm{m}(\mathbf{f})$ 


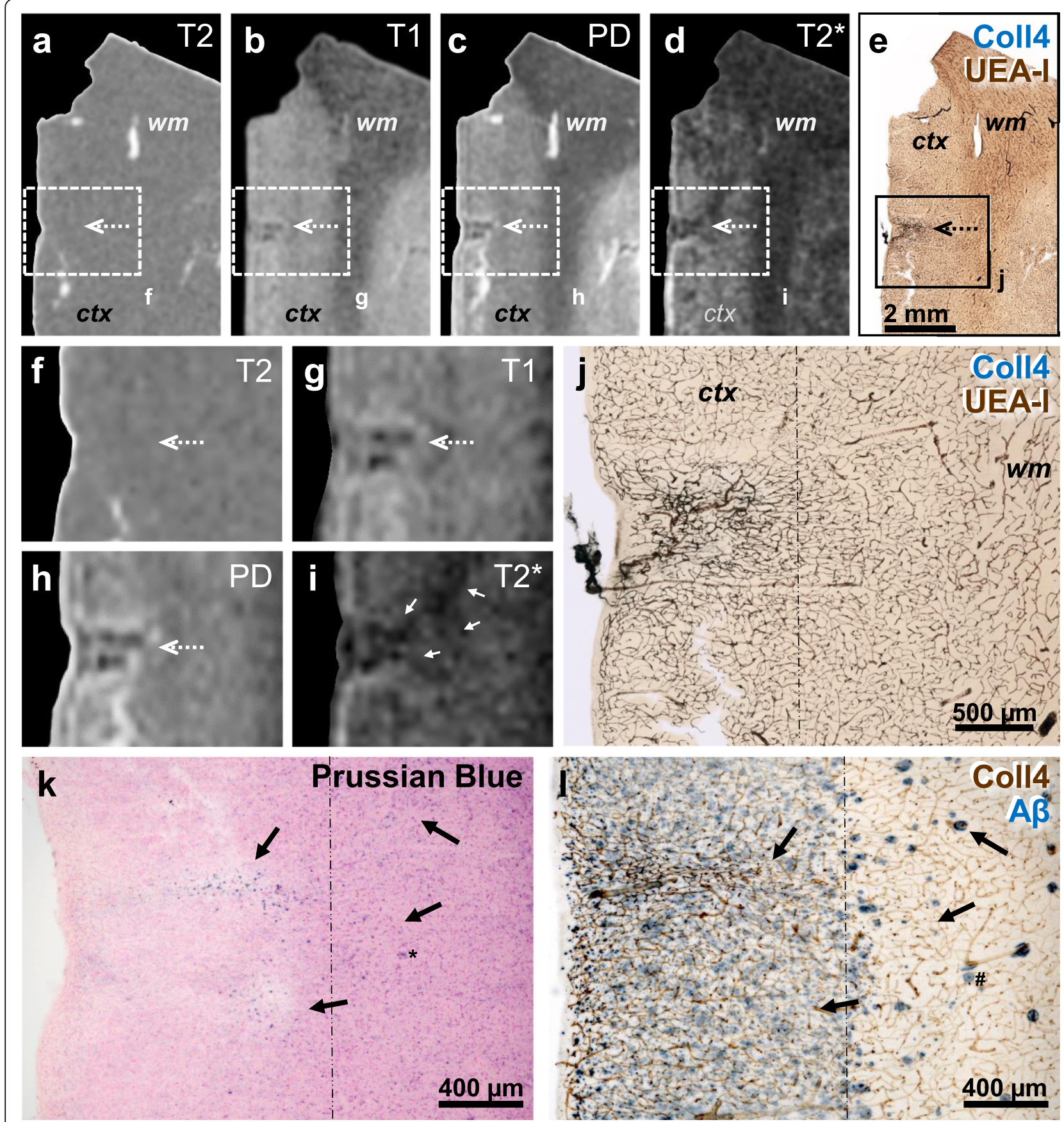

Fig. 9 Thick brain section (case 8) first scanned with 11.7T MRI and then double-labeled for Coll4 and UEA-I (a-j), whereas adjacent sections were stained with iron histochemistry (k; Prussian blue stain) and double-label immunohistochemistry for Coll4 and Aß (I). a, $\mathbf{f}$ T2-weighted image does not show changes in the signal intensity in the microinfarct area (dotted arrow) despite the strong hyperintense signal produced by saline in a tear nearby and around the section. $\mathbf{b}, \mathbf{g}$ The same microinfarct (dotted arrow) gave a hypointense T1-weighted signal. $\mathbf{c}, \mathbf{h}$ In the PD-weighted image, the microinfarct gave a reduced and its margin a hyperintense proton signal. $\mathbf{d}$-e and $\mathbf{i}$-j The hypointense microinfarct zone with a blooming effect in the T2*-weighted image (d, i) contained a dense microvascular meshwork (e, j). $\mathbf{k}$-I Microinfarct area giving a hypointense T2* signal (white arrows) showed iron deposition in the adjacent section (black arrows) independently from the diffuse AB deposition in superficial cortical layers, although larger iron aggregates in deep cortical layers (asterisk in $\mathbf{k}$ ) may be localized in cored A $\beta$ plaques (hashtag in I). ctx cortex; wm white matter. Scale bars: $2 \mathrm{~mm}(\mathbf{e}), 500 \mu \mathrm{m}(\mathbf{j})$ and $400 \mu \mathrm{m}(\mathbf{k}-\mathbf{l})$ 

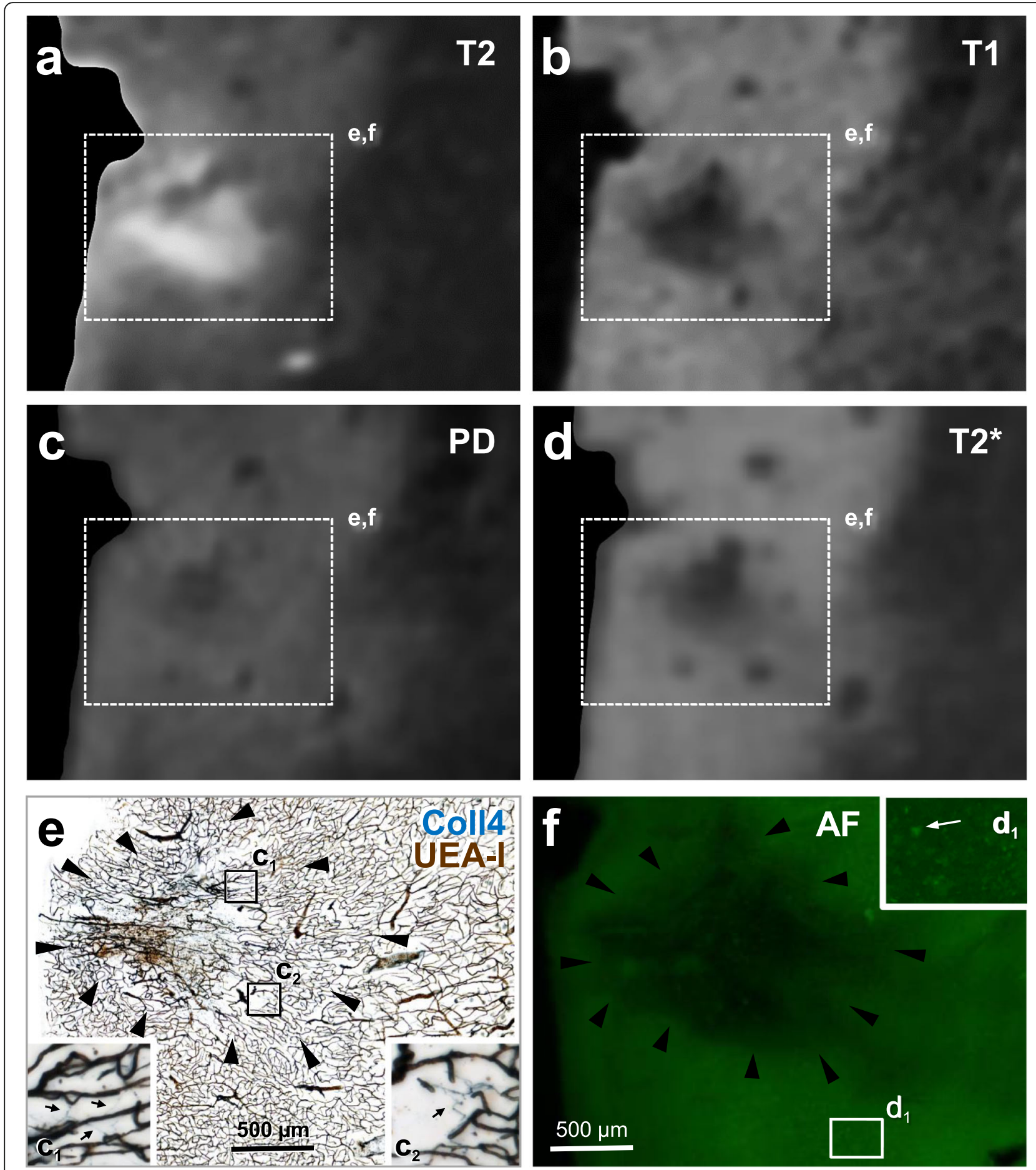

Fig. 10 Images from a $100 \mu$ m-thick brain section with a superficial cavitated microinfarct in the motor cortex (case 1). The section was first scanned with 11.7 T MRI (a-d) and then double-labeled for Coll4 and UEA-I (e) after capturing autofluorescence (f). a The cavity of the microinfarct gave a hyperintense signal in the T2-weighted image. $\mathbf{b}$ The T1-weighted MRI signal was hypointense in the microinfarct cavity and in tissue areas that showed thinning. c The PD-weighted image exhibited a hypointense signal in the core and a slightly hyperintense signal in the rim of the microinfarct cavity. $\mathbf{d}$ In the T2*-weighted image, large vessels and the microbleed in the cavity produced magnified hypointense signals (blooming effect). e The microinfarct cavity contained damaged microvessels and the rim of the cavity was rich in Coll4-positive string vessels that lacked UEA-I staining. $\mathbf{f}$ Autofluorescence (AF) of the tissue was reduced in the microinfarct cavity and in areas with tissue thinning around the cavity. Betz cells could also be identified based on their autofluorescence (arrow in inset $\mathbf{d}_{\mathbf{1}}$ ). Scale bars: $500 \mu \mathrm{m}$ (e-f) 
with a low iron load produced a low signal contrast (Fig. 8 , Supplementary file S3). In microinfarcts with iron accumulation, the intensity of the hypointense $\mathrm{T} 2 \%$-weighted MRI signal was higher in microinfarcts with a higher iron load when compared to microinfarcts with low iron accumulation, as can be seen in two different microinfarcts from the same case (Fig. 9 and Supplementary file S4).

\section{Discussion}

The microvasculature at the infarction zone of chronic cortical cerebral microinfarcts showed unique pathologic features that seemed to be largely independent of their location in different layers of the cerebral cortex. Common to all chronic microinfarcts identified in this study was tissue pallor with loss of neurons and lipofuscin pigment accumulation that was accompanied by a dense microvascular meshwork. The microvascular meshwork in the microinfarct core and the microinfarct margin contained a high density of string vessels that lacked expression of the endothelial marker UEA-l. In addition, the microinfarct core was infiltrated by activated CD68-positive macrophages and GFAP-positive astrocytes, whereas astrocytes often extended to the microinfarct periphery. Nevertheless, chronic cortical cerebral microinfarcts also showed some variability, especially with regard to their size and shape and the degree of iron accumulation in the microinfarct core, which impacted their detectability in postmortem ultra-high-resolution MRI.

These features of chronic cortical microinfarcts are consistent with findings obtained in thin paraffin sections stained for IHC and/or H\&E, which displayed pallor due to reduced eosinophilic staining of the neuropil and loss of neurons, astrogliosis, activated microglial cells, and occasionally cystic formations $[12,15,26]$. (Sub)acute cortical cerebral infarcts did not exhibit these pathological changes or a dense microvascular meshwork, thereby supporting the chronic nature of the microinfarcts imaged here [13, 33, 34]. Therefore, these microinfarcts were summarized as "chronic" microinfarcts now, although the observed pathologies may represent different phases of microinfarcts. In patients with stroke, where the sequence of pathological events in infarcts could be established based on the timepoint of injury, three phases were described: (1) Acute neuronal injury with cytoplasmic vacuolation, eosinophilia (red neurons), and ghost cell formation together with edematous spongiosis, (2) necrosis and organization, which was characterized initially by (a) acute inflammation with neutrophils, and later by (b) chronic inflammation with macrophages and perivascular cuffing, and finally (3) chronic resorption with macrophages, pallor, spongiosis and/or cavitation. Phases 2 and 3 also exhibited various degrees of axonal spheroids, gemistocytes, and neovascularization [34].

Larger and/or cavitated cortical cerebral microinfarcts were detectable in histological sections in the PN stain, Coll4-IHC, iron histochemistry and H\&E staining adapted to thick brain sections. However, smaller microinfarcts with minor tissue thinning in the absence of cavitation or puckering were often barely noticeable in thick H\&E-stained sections. Therefore, thick brain sections stained for PN stain and Coll4-IHC were screened for a more reliable identification and characterization of the latter microinfarcts. Microinfarcts are common in patients with mild cognitive impairment and dementia, but current estimates of their numbers in the human brain are largely based on the extrapolation of findings obtained in standard paraffin sections $[12,48]$. Therefore, the insights gained here into the organization of smaller cortical cerebral microinfarcts provide a basis for a more systematic investigation of cortical microinfarcts in larger patient cohorts. Our analyses of thick brain sections further allowed the reliable detection of comparatively small iron depositions in microinfarcts, although factors that influenced iron accumulation in chronic microinfarcts remained elusive. At chronic phases of microinfarcts, activated macrophages can persist in the core of the infarction zone, as shown here and by others [42, 50], where they may engulf extracellular lipofuscin aggregates resulting from neuronal cell death [8] or take up iron in response to neuroinflammatory processes [39]. Moreover, the microinfarct zone containing iron accumulation coincided with the zone infiltrated by activated microglial cells and a dense microvascular meshwork. Inasmuch as these cortical microinfarcts might have originally evolved from ischemic $[2,32]$ or hemorrhagic infarcts, a major source of iron in microbleeds is hemoglobin and its degradation products, which lead to transformation of macrophages into hemosiderophages [18, 31].

On postmortem MR images obtained with a field strength of 1 Tesla (T), only $25 \%$ of cortical microinfarcts were detected correctly and $25 \%$ were thought to be artifacts, whereas $33 \%$ of the histologically verified microinfarcts were not visible [19]. The detection rate of cortical microinfarcts was also improved considerably by imaging at $7 \mathrm{~T}$ in comparison to $3 \mathrm{~T}$, which was confirmed histologically through subsequent serial sectioning. Therefore, technical limitations, such as field strength, image resolution, pulsesequence parameters and MRI-histological matching were considered to be the major sources for obtaining false negative results $[15,25,47,49]$. However, our analyses with a field strength of $11.7 \mathrm{~T}$ now showed that there is also some variability in the histopathological features of cortical cerebral microinfarcts, including not only their size and shape, the cortical layer in which they occur, thinning, and cavitation, but also the degree of iron accumulation, which influenced MRI signals and the detectability of microinfarcts in this study. While microinfarcts produced hypointense MRI signals in T1-weighted, and to some extend also in PDweighted images, hyperintense $\mathrm{T} 2$ signals were more variable. Moreover, the T1 signal strength was more prominent when thinning of tissue or cavitation was evident, and 
strong T2 signals were also observed in microinfarcts with cavitation. These MRI signal intensities and contrasts produced by cavitated and non-cavitated cortical cerebral microinfarcts at $11.7 \mathrm{~T}$ in T1-, T2, PD- and T2*-weighted sequences were consistent with data obtained at $7 \mathrm{~T}$ in previous MRI-histological correlation studies $[15,46,49]$. However, MRI signals of smaller and narrower microinfarcts with less tissue thinning could be also imaged and histologically studied here with $11.7 \mathrm{~T}$ MRI, although the MRI signals produced by these microinfarcts with lower field strengths or in vivo MRI scans remains to be determined. Iron accumulation, histologically confirmed with Prussian blue staining, was the likely source of changes in susceptibility detected in chronic microinfarcts in our T2*-weighted MR images. In clinical studies, T2*-weighted MRI sequences are commonly used to detect microbleeds in patients with CAA [53]. Of note, several cases here suffered from cerebrovascular diseases resulting in stroke and most of the individuals either lacked extracellular $A \beta$ aggregates or they displayed mainly parenchymal $A \beta$ deposits.

A major obstacle in analyses of structural correlates of MRI signals is the identification of the spatial correspondence of a lesion between the MR images and histological sections, commonly referred to as MRI-histological matching $[1,37]$. Moreover, histological sections often show deformations that occur during resection, handling and histological processing of the tissue, making the registration of tissue-derived histological images to the MR images necessary. During registration, deformations in histological images are removed with algorithms used for remodeling and transformation of the images with the goal to improve the spatial correspondence between MRI scans and histological images [24, 37]. By imaging free-floating thick brain sections with a field strength of $11.7 \mathrm{~T}$, we could not only obtain MR images with ultra-high resolution here, but also circumvent problems associated with MRI-histological matching and image registration. Furthermore, we could achieve a direct correlation between the MRI signals acquired and histological changes in the microinfarction zone including smaller iron deposits and string vessels that are difficult to detect in standard paraffin sections. Nevertheless, ultra-high-resolution imaging of $100 \mu \mathrm{m}$ thick brain sections might result in a reduced MRI signal-to-noise ratio despite the use of high field strengths. Postmortem formalin fixation also alters MRI signals [36], potentially affecting the detection of structural changes in the density of microvessels, macrophages or astrocytes, especially in T1- and T2-weighted and PD images.

The blood supply of the cerebral cortex is provided by branches of major cerebral arteries that are located in the subarachnoid space. Arterioles arising from these branches enter gyri and sulci in a perpendicular fashion and give rise to branches that supply specific layers of the cerebral cortex. Duvernoy et al. [17] have classified cortical arterioles from
A1-A6 based on the depth of the cortical layer they reach, which correlates well with the locations of the microinfarcts observed here. Thus, hypoperfusion or bleeding in the zones supplied by A1-A5 type arterioles or their branches is perfectly suited to cause circumscribed infarctions in the superficial and deep layers of the cerebral cortex. Because A5 type arterioles run through the cortex and reach the subcortical white matter, their occlusion may result in transcortical microinfarctions. Moreover, portions of A5 and A6 arterioles that reach the subcortical white matter form multiple anastomoses (Duvernoy at al., 1981). This may explain the confinement of microinfarcts to deep cortical and juxtacortical locations, and the sparing of the deeper subcortical white matter, when juxtacortical arteriolar branches are affected. Rupture or occlusion of these arterioles could be caused by CAA or atherosclerosis of leptomeningeal vessels and their arteriolar branches that supply the cerebral cortex [31, 43]. Other causes of cortical cerebral microinfarcts include thrombotic microembolisms originating from the heart (e.g., endocarditis) or plaques in cerebral arteries, lipid microembolisms following major surgeries, hereditary forms of small vessel disease such as CADASIL, leukoencephalopathy, and radiation therapy $[11,25]$.

A common feature of the chronic cortical cerebral microinfarcts seen here was the presence of a dense microvascular meshwork with string vessels in the infarction zone. String vessels are known to emerge during the course of vascular regression, which is tightly regulated during angiogenesis and neovascularization [30,38, 51]. Development of string vessels can be also mediated by a loss of factors that promote endothelial cell survival and angiogenesis such as vascular endothelial growth factor (VEGF) [3, 10, 40]. In our cases, Coll4-positive string vessels at microinfarcts and their periphery lacked UEA-l expression indicating endothelial recession, which is associated with cessation of blood perfusion in the vessels affected [10, 22, 55]. Thus, the presence of string vessels pointed to disturbances in the microcirculation in and around cortical cerebral microinfarcts, which may explain the comparatively strong MRI signals obtained from relatively small cortical microinfarcts in vivo [27, 28, 32]. Thus, advanced techniques that detect changes in cerebral perfusion [20] and/ or axonal disorganization [14] may further enhance the visualization of chronic cortical cerebral microinfarcts with in vivo MRI, especially when combined with techniques that use local perturbations of the MR phase to enhance T2* contrast, e.g., 3D T2*-weighted imaging with postprocessing susceptibility-weighted imaging [25], to distinguish microbleeds from calcifications.

\section{Conclusions}

Our results indicate that chronic cortical cerebral microinfarctions occur at superficial, middle and deep layers of the cortex, and they can extend throughout all 
cortical layers, which is consistent with the pattern of blood supply provided by different types of cortical arterioles. Ultra-high resolution MRI performed in single sections permits the characterization of MRI signal contrast in small chronic cortical cerebral microinfarcts with minor thinning. Together with recent improvements in the resolution of imaging techniques in vivo, the knowledge of the location, shape and MRI signal contrast of cortical cerebral microinfarcts and patterns of iron accumulation may aid in the development of new analytical tools for their identification with imaging. Moreover, cortical cerebral microinfarcts at chronic stages exhibit a high vessel density with numerous string vessels in and near the microinfarct, which may alter the pattern of blood flow in the infarction zone. Such changes in the microcirculation in the microinfarction zone might be detectable with advanced imaging techniques, making it possible to identify cortical cerebral microinfarcts in living patients.

\section{Supplementary information}

Supplementary information accompanies this paper at https://doi.org/10. 1186/s40478-020-00900-1.

Additional file 1: Figure S1. A chronic cortical cerebral microinfarct in the superior frontal gyrus with minor tissue thinning that is located in mid-cortical layers (case 6) is shown in two neighboring $100 \mu$ m-thick brain sections stained with the pigment NissI (PN) (a) and modified H\&E stains $(\mathbf{b}-\mathbf{c})$. In thick brain sections, the boundaries of the pale microinfarct are clearly evident in the PN stain, but the paleness of the microinfarction zone is more difficult to discern in the modified H\&E stain. The inset (c) shows an enlaged area from (b) with various cell types and vessels (arrow) in the core of the microinfarct stained with H\&E. Scale bars: $400 \mu \mathrm{m}(\mathbf{a}-\mathbf{b})$ and $150 \mu \mathrm{m}(\mathbf{c})$

Additional file 2.

Additional file 3: Figure S3. The insets of MR images from Fig. 8 are presented here again (a-d) together with an autofluorescence image (AF) taken from the same $100 \mu$ m-thick section after the MRI scan (i). The microinfarct was also visualized in neighboring sections using singlelabeling for Coll4 (e), Prussian blue staining (f), double-labeling for Coll4 and beta amyloid (AB) (g) and the pigment Nissl (PN) stain (h).

Additional file 4: Figure S4. MRI scans and histology of a second microinfarct from the same imaged (a-j) and same adjacent sections (case 8) shown in Fig. $8(\mathbf{k}, \mathbf{l})$. Although this microinfarct also contains a dense Coll4- and UEA-I-positive microvascular meshwork $(\mathbf{e}, \mathbf{j})$ and cortical $A \beta$ deposit (diffuse superficially, cored in deep layers; $\mathbf{I}$ ), its signal in T1-, T2- and PD-weighted images is difficult to detect (a-c and f-h). However, the T2*-weighted hypointense signal produced by the microinfarction zone is comparatively weak $(\mathbf{d}, \mathbf{i})$, which correlates well with the lower degree of iron accumulation at the microinfarction area (k) compared to the other microinfarct. Scale bars: $2 \mathrm{~mm}(\mathbf{e})$ and $500 \mu \mathrm{m}(\mathbf{j}-\mathbf{I})$.

\section{Abbreviations}

3D: Three-dimensional; ABC: Avidin-biotin-peroxidase complex;

AD: Alzheimer's disease; BSA: Bovine serum albumin; CAA: Cerebral amyloid angiopathy; CDR: Clinical Dementia Rating; COLL4: Collagen 4; DAB: Diaminobenzidine tetrahydrochloride; GFAP: Glial fibrillary acidic protein; H\&E: Hematoxylin eosin; ICH: Immunohistochemistry; MMSE: Mini Mental Status Examination; MR: Magnetic resonance; MRI: Magnetic resonance imaging; NFT: Neurofibrillary changes; NSA: Number of signal averages; PD: Proton density; PEG: Polyethylene glycol; PN: Pigment Nissl stain; TBS: Tris-buffered saline; TE: Echo time; TR: Repetition time; UEA-I: Ulex europaeus lectin

\section{Acknowledgements}

Ms. Gabriele Ehmke, Ms. Siegrid Baumann and Ms. Patricia Häring are acknowledged for expert technical assistance.

\section{Authors' contributions}

DYH, JK and HB conceived the study. NH, KA, JM, ACL and JK characterized the patients. AS, JW, HB, KDT and DYH performed neuropathological assessments. DYH, KDT and HB developed histological procedures in thick brain sections. DYH, TM, KMEF, HPM and VR adapted MRI acquisition protocols to postmortem imaging with the 11.7T MRI scanner. DYH, TM, AA and VR established postmortem ultra-high-resolution MRI of single-sections. DYH and TM performed single section MRI-histopathological correlations and analyzed the data. DYH wrote the manuscript. All authors read and approved the final manuscript

\section{Funding}

The project was funded by the Corona Stiftung Germany, through intramural funds to DY-H., and in part, by the Foundation for Research on Alzheimer Disease (FRA), Paris (HB)

Availability of data and materials

All data generated or analyzed during this study are included in this published article.

\section{Ethics approval and consent to participate}

Brains were donated to the Brain Bank at the University of Ulm with the consent of the patients or their surrogates in accordance with the Declaration of Helsinki. The research was approved by the University of UIm Ethics Committee on Human Research.

\section{Consent for publication}

Not applicable.

\section{Competing interests}

The authors declare that they have no competing interests.

\section{Author details}

${ }^{1}$ Clinical Neuroanatomy, Department of Neurology, Institute for Biomedical Research, Ulm University, Helmholtzstr. 8/1, 89081 Ulm, Germany. ${ }^{2}$ Neurology Clinic, Department of Neurology, School of Medicine, Ulm University, Ulm, Germany. ${ }^{3}$ Core Facility Small Animal MRI, Medical Faculty, Ulm University, Ulm, Germany. ${ }^{4}$ Department of Internal Medicine II, University Hospital UIm, Ulm, Germany. ${ }^{5}$ Department of Pathology, Section Neuropathology, University Hospital Ulm, Ulm, Germany. ${ }^{6}$ Institute of Neuropathology, RWTH Aachen University Hospital, Aachen, Germany. ${ }^{7}$ Department of Medical Biosciences \& Pathology, Umeå Universitet, Umeå, Sweden.

Received: 25 December 2019 Accepted: 21 February 2020

Published online: 13 March 2020

\section{References}

1. Absinta M, Nair G, Filippi M, Ray-Chaudhury A, Reyes-Mantilla Ml, Pardo CA et al (2014) Postmortem magnetic resonance imaging to guide the pathologic cut: individualized, 3-dimensionally printed cutting boxes for fixed brains. J Neuropathol Exp Neurol 73:780-788. https://doi.org/10.1097/ NEN.00000000000000096

2. Arvanitakis Z, Capuano AW, Leurgans SE, Buchman AS, Bennett DA, Schneider JA (2017) The relationship of cerebral vessel pathology to brain microinfarcts. Brain Pathol 27:77-85. https://doi.org/10.1111/bpa.12365

3. Baffert F, Le T, Sennino B, Thurston G, Kuo CJ, Hu-Lowe D et al (2006) Cellular changes in normal blood capillaries undergoing regression after inhibition of VEGF signaling. Am J Physiol Heart Circ Physiol 290:H547-H559. https://doi.org/10.1152/ajpheart.00616.2005

4. Braak H (1976) On the striate area of the human isocortex. A Golgi- and pigmentarchitectonic study. J Comp Neurol 166:341-364. https://doi.org/10. 1002/cne.901660305 
5. Braak H, Braak E (1991) Demonstration of amyloid deposits and neurofibrillary changes in whole brain sections. Brain Pathol 1:213-216. https://doi.org/10.1111/j.1750-3639.1991.tb00661.x

6. Braak H, Braak E (1991) Neuropathological stageing of Alzheimer-related changes. Acta Neuropathol 82:239-259

7. Braak H, Del Tredici K, Rub U, de Vos RA, Jansen Steur EN, Braak E (2003) Staging of brain pathology related to sporadic Parkinson's disease. Neurobiol Aging 24:197-211

8. Braak H, Feldengut S, Kassubek J, Yilmazer-Hanke D, Del Tredici K (2018) Two histological methods for recognition and study of cortical microinfarcts in thick sections. Eur J Histochem 62. https://doi.org/10.4081/ejh.2018.2989

9. Brenowitz WD, Hubbard RA, Keene CD, Hawes SE, Longstreth WT Jr, Woltjer RL et al (2017) Mixed neuropathologies and associations with domainspecific cognitive decline. Neurology 89:1773-1781. https://doi.org/10.1212/ WNL.0000000000004567

10. Brown WR (2010) A review of string vessels or collapsed, empty basement membrane tubes. J Alzheimers Dis 21:725-739. https://doi.org/10.3233/JAD2010-100219

11. Brown WR, Thore CR (2011) Review: cerebral microvascular pathology in ageing and neurodegeneration. Neuropathol Appl Neurobiol 37:56-74. https://doi.org/10.1111/j.1365-2990.2010.01139.x

12. Brundel M, de Bresser J, van Dillen JJ, Kappelle LJ, Biessels GJ (2012) Cerebral microinfarcts: a systematic review of neuropathological studies. J Cereb Blood Flow Metab 32:425-436. https://doi.org/10.1038/jcbfm.2011.200

13. Chuaqui R, Tapia J (1993) Histologic assessment of the age of recent brain infarcts in man. J Neuropathol Exp Neurol 52:481-489. https://doi.org/10. 1097/00005072-199309000-00006

14. Coban H, Tung S, Yoo B, Vinters HV, Hinman JD (2017) Molecular disorganization of axons adjacent to human cortical microinfarcts. Front Neurol 8:405. https://doi.org/10.3389/fneur.2017.00405

15. De Reuck J, Deramecourt V, Auger F, Durieux N, Cordonnier C, Devos D et al (2014) Post-mortem 7.0-tesla magnetic resonance study of cortical microinfarcts in neurodegenerative diseases and vascular dementia with neuropathological correlates. J Neurol Sci 346:85-89. https://doi.org/10. 1016/j.jns.2014.07.061

16. Del Tredici K, Braak H (2012) Spinal cord lesions in sporadic Parkinson's disease Acta Neuropathol 124:643-664. https://doi.org/10.1007/s00401-012-1028-y

17. Duvernoy HM, Delon S, Vannson JL (1981) Cortical blood vessels of the human brain. Brain Res Bull 7:519-579

18. Fazekas F, Kleinert R, Roob G, Kleinert G, Kapeller P, Schmidt R et al (1999) Histopathologic analysis of foci of signal loss on gradient-echo T2* weighted MR images in patients with spontaneous intracerebra hemorrhage: evidence of microangiopathy-related microbleeds. AJNR Am J Neuroradiol 20:637-642

19. Fernando MS, O'Brien JT, Perry RH, English P, Forster G, Mc Meekin W et al (2004) Comparison of the pathology of cerebral white matter with postmortem magnetic resonance imaging (MRI) in the elderly brain. Neuropathol Appl Neurobiol 30:385-395. https://doi.org/10.1111/j.13652990.2004.00550.x

20. Ferro DA, Mutsaerts HJ, Hilal S, Kuijf HJ, Petersen ET, Petr J et al (2019) Cortical microinfarcts in memory clinic patients are associated with reduced cerebral perfusion. J Cereb Blood Flow Metab. https://doi.org/10.1177/ $0271678 \times 19877403$

21. Ferro DA, van Veluw SJ, Koek HL, Exalto LG, Biessels GJ, Utrecht Vascular Cognitive Impairment study g (2017) Cortical cerebral microinfarcts on 3 tesla MRI in patients with vascular cognitive impairment. J Alzheimers Dis 60:1443-1450. https://doi.org/10.3233/JAD-170481

22. Forsberg KME, Zhang $Y$, Reiners $J$, Ander $M$, Niedermayer $A$, Fang $L$ et al (2018) Endothelial damage, vascular bagging and remodeling of the microvascular bed in human microangiopathy with deep white matter lesions. Acta Neuropathol Commun 6:128. https://doi.org/10.1186/s40478018-0632-z

23. Gallyas F (1971) Silver staining of Alzheimer's neurofibrillary changes by means of physical development. Acta Morphol Acad Sci Hung 19:1-8

24. Goubran M, Crukley C, de Ribaupierre S, Peters TM, Khan AR (2013) Image registration of ex-vivo MRI to sparsely sectioned histology of hippocampal and neocortical temporal lobe specimens. Neuroimage 83:770-781. https://doi.org/10.1016/j.neuroimage.2013.07.053

25. Haller S, Vernooij MW, Kuijer JPA, Larsson EM, Jager HR, Barkhof F (2018) Cerebral microbleeds: imaging and clinical significance. Radiology 287:1128. https://doi.org/10.1148/radiol.2018170803
26. Ince PG, Minett T, Forster G, Brayne C, Wharton SB, Medical Research Council Cognitive F et al (2017) Microinfarcts in an older populationrepresentative brain donor cohort (MRC CFAS): prevalence, relation to dementia and mobility, and implications for the evaluation of cerebral small vessel disease. Neuropathol Appl Neurobiol 43:409-418. https://doi.org/10. $1111 /$ nan.12363

27. Ishikawa H, li Y, Niwa A, Shindo A, Ito A, Matsuura K et al (2018) Comparison of Premortem magnetic resonance imaging and postmortem autopsy findings of a cortical microinfarct. J Stroke Cerebrovasc Dis 27:2623-2626. https://doi.org/10.1016/j.jstrokecerebrovasdis.2018.05.037

28. Jouvent E, Poupon C, Gray F, Paquet C, Mangin JF, Le Bihan D et al (2011) Intracortical infarcts in small vessel disease: a combined 7-T postmortem MRI and neuropathological case study in cerebral autosomal-dominant arteriopathy with subcortical infarcts and leukoencephalopathy. Stroke 42: e27-e30. https://doi.org/10.1161/STROKEAHA.110.594218

29. Kapasi A, Leurgans SE, James BD, Boyle PA, Arvanitakis Z, Nag S et al (2018) Watershed microinfarct pathology and cognition in older persons. Neurobiol Aging 70:10-17. https://doi.org/10.1016/j.neurobiolaging.2018.05.027

30. Kerr BA, West XZ, Kim YW, Zhao Y, Tischenko M, Cull RM et al (2016) Stability and function of adult vasculature is sustained by Akt/Jagged1 signalling axis in endothelium. Nat Commun 7:10960. https://doi.org/10. 1038/ncomms10960

31. Kövari E, Herrmann FR, Hof PR, Bouras C (2013) The relationship between cerebral amyloid angiopathy and cortical microinfarcts in brain ageing and Alzheimer's disease. Neuropathol Appl Neurobiol 39:498-509. https://doi. org/10.1111/nan.12003

32. Leng $X$, Fang H, Pu Y, Zou X, Pan Y, Soo YOY et al (2017) Cortical microinfarcts in patients with middle cerebral artery stenosis. J Stroke Cerebrovasc Dis 26:1760-1765. https://doi.org/10.1016/j.jstrokecerebrovasdis. 2017.04.002

33. Margaritescu O, Mogoanta L, Pirici I, Pirici D, Cernea D, Margaritescu C (2009) Histopathological changes in acute ischemic stroke. Romanian J Morphol Embryol 50:327-339

34. Mena H, Cadavid D, Rushing EJ (2004) Human cerebral infarct: a proposed histopathologic classification based on 137 cases. Acta Neuropathol 108 524-530. https://doi.org/10.1007/s00401-004-0918-z

35. Mulisch M, Welsch U (2015) Romeis - Mikroskopische Technik, 19th edn. Wiesbaden: Springer Spektrum (Verlag)

36. Pfefferbaum A, Sullivan EV, Adalsteinsson E, Garrick T, Harper C (2004) Postmortem MR imaging of formalin-fixed human brain. Neuroimage 21: 1585-1595. https://doi.org/10.1016/j.neuroimage.2003.11.024

37. Pichat J, Iglesias JE, Yousry T, Ourselin S, Modat M (2018) A survey of methods for 3D histology reconstruction. Med Image Anal 46:73-105. https://doi.org/10.1016/j.media.2018.02.004

38. Poche RA, Hsu CW, McElwee ML, Burns AR, Dickinson ME (2015) Macrophages engulf endothelial cell membrane particles preceding pupillary membrane capillary regression. Dev Biol 403:30-42. https://doi.org/ 10.1016/j.ydbio.2015.03.017

39. Rathnasamy G, Ling EA, Kaur C (2013) Consequences of iron accumulation in microglia and its implications in neuropathological conditions. CNS Neurol Disord Drug Targets 12:785-798. https://doi.org/10.2174/ 18715273113126660169

40. Reeson P, Choi K, Brown CE (2018) VEGF signaling regulates the fate of obstructed capillaries in mouse cortex. Elife 7. https://doi.org/10.7554/eLife. 33670

41. Shatil AS, Matsuda KM, Figley CR (2016) A method for whole brain ex vivo magnetic resonance imaging with minimal susceptibility artifacts. Front Neurol 7:208. https://doi.org/10.3389/fneur.2016.00208

42. Smith EE, Schneider JA, Wardlaw JM, Greenberg SM (2012) Cerebral microinfarcts: the invisible lesions. Lancet Neurol 11:272-282. https://doi. org/10.1016/S1474-4422(11)70307-6

43. Thal DR, Grinberg LT, Attems J (2012) Vascular dementia: different forms of vessel disorders contribute to the development of dementia in the elderly brain. Exp Gerontol 47:816-824. https://doi.org/10.1016/j. exger.2012.05.023

44. Urban S, Yilmazer-Hanke DM (1999) The pigmentarchitectonic divisions and neuronal types of the central nucleus and intercalated masses of the human amygdala. J Hirnforsch 39:311-319

45. van Rooden S, Goos JD, van Opstal AM, Versluis MJ, Webb AG, Blauw GJ et al (2014) Increased number of microinfarcts in Alzheimer disease at 7-T MR imaging. Radiology 270:205-211. https://doi.org/10.1148/radiol.13130743 
46. van Veluw SJ, Charidimou A, van der Kouwe AJ, Lauer A, Reijmer YD, Costantino I et al (2016) Microbleed and microinfarct detection in amyloid angiopathy: a high-resolution MRI-histopathology study. Brain 139:31513162. https://doi.org/10.1093/brain/aww229

47. van Veluw SJ, Hilal S, Kuijf HJ, Ikram MK, Xin X, Yeow TB et al (2015) Cortical microinfarcts on $3 \mathrm{~T}$ MRI: clinical correlates in memory-clinic patients. Alzheimers Dement 11:1500-1509. https://doi.org/10.1016/j.jalz.2014.12.010

48. van Veluw SJ, Shih AY, Smith EE, Chen C, Schneider JA, Wardlaw JM et al (2017) Detection, risk factors, and functional consequences of cerebral microinfarcts. Lancet Neurol 16:730-740. https://doi.org/10.1016/S14744422(17)30196-5

49. van Veluw SJ, Zwanenburg JJ, Rozemuller AJ, Luijten PR, Spliet WG, Biessels GJ (2015) The spectrum of MR detectable cortical microinfarcts: a classification study with 7-tesla postmortem MRI and histopathology. J Cereb Blood Flow Metab 35:676-683. https://doi.org/10.1038/jcbfm.2014.258

50. Wang M, lliff JJ, Liao Y, Chen MJ, Shinseki MS, Venkataraman A et al (2012) Cognitive deficits and delayed neuronal loss in a mouse model of multiple microinfarcts. J Neurosci 32:17948-17960. https://doi.org/10.1523/ JNEUROSCI.1860-12.2012

51. Watson EC, Grant ZL, Coultas L (2017) Endothelial cell apoptosis in angiogenesis and vessel regression. Cell Mol Life Sci 74:4387-4403. https://doi.org/10.1007/s00018-017-2577-y

52. Westover MB, Bianchi MT, Yang C, Schneider JA, Greenberg SM (2013) Estimating cerebral microinfarct burden from autopsy samples. Neurology 80:1365-1369. https://doi.org/10.1212/WNL.0b013e31828c2f52

53. Yamada M (2015) Cerebral amyloid angiopathy: emerging concepts. J Stroke 17:17-30. https://doi.org/10.5853/jos.2015.17.1.17

54. Yilmazer-Hanke DM, Wolf HK, Schramm J, Elger CE, Wiestler OD, Blumcke I (2000) Subregional pathology of the amygdala complex and entorhinal region in surgical specimens from patients with pharmacoresistant temporal lobe epilepsy. J Neuropathol Exp Neurol 59:907-920. https://doi. org/10.1093/jnen/59.10.907

55. Zille M, Ikhsan M, Jiang Y, Lampe J, Wenzel J, Schwaninger M (2019) The impact of endothelial cell death in the brain and its role after stroke: a systematic review. Cell Stress 3:330-347. https://doi.org/10.15698/cst2019.11.203

\section{Publisher's Note}

Springer Nature remains neutral with regard to jurisdictional claims in published maps and institutional affiliations.

Ready to submit your research? Choose BMC and benefit from:

- fast, convenient online submission

- thorough peer review by experienced researchers in your field

- rapid publication on acceptance

- support for research data, including large and complex data types

- gold Open Access which fosters wider collaboration and increased citations

- maximum visibility for your research: over $100 \mathrm{M}$ website views per year

At $\mathrm{BMC}$, research is always in progress.

Learn more biomedcentral.com/submissions 